\title{
Synthesis of Mechanisms by Methods of Nonlinear Dynamics
}

\author{
Michael Valášek, Zbyněk Šika \\ Department of Mechanics, Biomechanics and Mechatronics Faculty of Mechanical Engineering Czech Technical University \\ in Prague Technická 4, 16607 Praha 6, Czech Republic \\ Corresponding author: Michael.Valasek@fs.cvut.cz
}

\begin{abstract}
This paper deals with a new method for parametric kinematic synthesis of mechanisms. The traditional synthesis procedure based on collocation, correction and optimization suffers from the local minima of objective functions, usually due to the local unassembled configurations which must be overcome. The new method uses the time varying values of the synthesized dimensions of the mechanism as if the mechanism had elastic links and guidances. The time varying dimensions form the basis for an accompanying nonlinear dynamical dissipative system and the synthesis is transformed into the time evolution of this accompanying dynamical system. Its dissipativity guarantees the termination of the synthesis. The synthesis always covers the parametric kinematic synthesis, but it can be advantageously extended into the optimization of any further criteria. The main advantage of the method described here for dealing with mechanism synthesis is that it overcomes the unassembled configurations of the synthesized mechanisms and enables any further synthesis criteria to be introduced, and terminates due to dissipation of the accompanied dynamical system.
\end{abstract}

Keywords: synthesis of mechanisms; time varying dimensions; evolution of dissipative systems; multi-objective optimization; dexterity; workspace; built-up space.

\section{Introduction}

Like other engineering problems, the parametric kinematic synthesis of mechanisms has profited from computational methods, e.g [1]. Traditional methods are described specifically for a particular type of mechanisms [2-5]. General iterative procedures based on various optimization methods $[1,6-11]$ have been developed recently. The currently used methods seem to be sufficiently powerful and able to find solutions for most problems in mechanism synthesis. However, all these methods suffer from two related problems. The first problem is that the dimensions of the mechanism that are being optimized do not allow the mechanism to be assembled in all the positions required for the desired motion. The second problem is that if some mechanism synthesis iteration fails for a certain parameter because of a constraint and/or an assembly violation, the whole knowledge from this iteration is lost. A solution to the first problem has been proposed with the use of time-varying dimensions during the dimension iteration process for the mechanism [12]. By allowing the dimensions of the system that are treated as the design variables to vary during the motion of the mechanism, it is possible to guarantee that the system can be assembled in all configurations. This leads to a variation of each dimension during the cycle of the mechanism. The synthesis problem is then solved by attempting to minimize the deviation from the mean value for all the design variables during the cycle. A solution to the second problem is proposed in [13], where the non-assembly positions are used for the synthesis. However, this approach suffers from a slow iteration process with unclear termination. This problem is overcome by the new method described in this paper. This new approach has been described in [14-17]. Our paper formulates the method in a specialized way for a very large but restricted class of synthesis problems of mechanisms. This enables the description to be made in a more precise, systematic and algorithmic way. In addition, two interesting examples are included that have not previously been fully described [17].

\section{General formulation of the method}

\subsection{Traditional vector method}

The initial assumption is that the mechanism to be synthesized can be analyzed by the vector method (for 2D problems e.g. [18], for 3D problems e.g. [19]). This is the only reduction in generality compared to the formulation in [14-17]. Let us further describe the general procedure without loss of generality just for $2 \mathrm{D}$.

The mechanism to be synthesized is described by the vector method, which leads to a description of the mechanism by vector polygons with vector vertices $V_{i}(i=1, \ldots, n)$ (a simple case is shown in Fig. 1) and vectors $\boldsymbol{b}_{\boldsymbol{i}}\left(i=1, \ldots, n_{V}, n_{V} \geq n\right)$ with the parameters the lengths $b_{i}$ and the angles $\beta_{i}$ of the vectors (Fig. 2). 


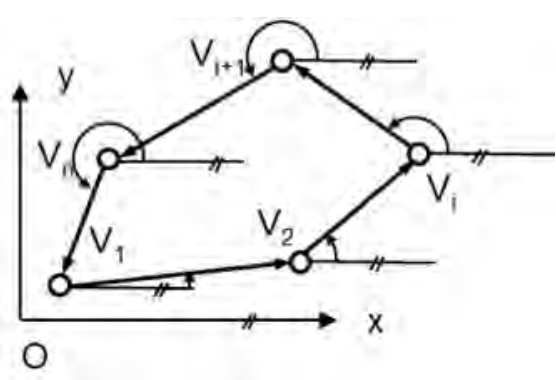

Figure 1: Simple vector polygon.

These parameters include both the coordinates (variable parameters from $b_{i}$ and $\beta_{i}$ ) and the dimensions (constant parameters from $b_{i}$ and $\beta_{i}$ ) of the synthesized mechanism. Parameters $b_{i}$ and $\beta_{i}$ can therefore be split into variable coordinates $s_{k}$ and constant parameters $p_{j}(j=1, \cdots, m)$. This is the traditional formulation of the mechanism synthesis where the dimensions being synthesized are constant values.

The fundamental objective functions are typically constructed on the network of mechanism positions within the desired workspace. Let index $r$ denote the general position of the mechanism, $r=1,2, \ldots, N$, where $N$ is the number of such representative positions, and the corresponding coordinates are $s_{j, r}$. Some of the coordinates $s_{j, r}$ are prescribed for particular positions $r=1,2, \ldots, N$. The traditional synthesis method is a search for constant parameters $p_{j}(j=1, \ldots, m)$ such that the closure conditions of the vector polygons in all $r(r=1,2, \ldots, N)$ positions are fulfilled [18-19].

\subsection{New method}

The new method is based on variation of the mechanism dimensions. They are no longer constant, and the time varying parameters $p_{j, r}$ can vary between the positions $r(r=1,2, \ldots, N)$ of the mechanism. During the synthesis process it is therefore admitted

$$
\begin{gathered}
p_{1,1} \neq p_{1,2} \neq \cdots \neq p_{1, N}, \\
p_{2,1} \neq p_{2,2} \neq \cdots \neq p_{2, N}, \\
\vdots \\
p_{m, 1} \neq p_{m, 2} \neq \cdots \neq p_{m, N}
\end{gathered}
$$

and the synthesis goal is to reach equality of all parameters at the end of the synthesis

$$
\begin{gathered}
p_{1,1} \cong p_{1,2} \cong \ldots \cong p_{1, N} \\
p_{2,1} \cong p_{2,2} \cong \ldots \cong p_{2, N} \\
\vdots \\
p_{m, 1} \cong p_{m, 2} \cong \ldots \cong p_{m, N}
\end{gathered}
$$

However, new coordinates are used by the new method. They are the Cartesian coordinates



Figure 2: Vector parameters.

$x_{V_{i, r}}, y_{V_{i, r}}$ of the polygon vector vertices $V_{i}(i=$ $1, \ldots, n)$, which are variable.

The varying values of parameters $p_{j, r}$ that correspond to the dimensions being synthesized and that are constant in the traditional vector method can be determined from the positions of the vertices $V_{i}$ in each position $r$. If the distance $V_{i} V_{i+1}$ corresponds to the constant length dimension of the synthesized mechanism, then its time varying value is computed in each position $r$ and each time

$$
p_{i, r}=b_{i, r}=\sqrt{\left(x_{V_{i+1, r}}-x_{V_{i, r}}\right)^{2}+\left(y_{V_{i+1, r}}-y_{V_{i, r}}\right)^{2}}
$$

and if angle $V_{i} V_{i+1}$ with respect to the frame corresponds to the constant length dimension of the synthesized mechanism then its time varying value is computed in each position $\mathrm{r}$ and each time

$$
p_{i, r}=\beta_{i, r}=\operatorname{atan} \frac{y_{V_{i+1, r}}-y_{V_{i, r}}}{x_{V_{i+1, r}}-x_{V_{i, r}}} .
$$

The new coordinates $x_{V_{i, r}}, y_{V_{i, r}}$ and the parameters $p_{j, r}$ are time varying and then constant at the synthesized mechanism after synthesis with the time varying values. The new coordinates $x_{V_{i, r}}, y_{V_{i, r}}$ are the coordinates of the accompanying nonlinear dynamical dissipative system, which is described by the Lagrange equations. Its kinetic energy is

$$
E_{\mathrm{k}}=\frac{1}{2} \sum_{k=1}^{n} \sum_{r=1}^{N} m_{k}\left(\dot{x}_{k, r}^{2}+\dot{y}_{k, r}^{2}\right),
$$

where $m_{k}$ are artificially introduced masses, and its potential energy is

$$
E_{\mathrm{p}}=\frac{1}{2} \sum_{k=1}^{n} \sum_{r=1}^{N} k_{k} \sum_{i=1}^{N}\left(p_{k, r}-p_{k, i}\right)^{2},
$$

where $k_{k}$ are artificially introduced stiffnesses. The potential energy describes the excitation of the new dynamic system whenever parameters $p_{k, r}$ are not equal to each other between the positions $r=1, \ldots, N$. The dissipation is introduced by the Raleigh function

$$
D=\frac{1}{2} \sum_{k=1}^{n} \sum_{r=1}^{N} B_{k}\left(\dot{x}_{k, r}^{2}+\dot{y}_{k, r}^{2}\right),
$$




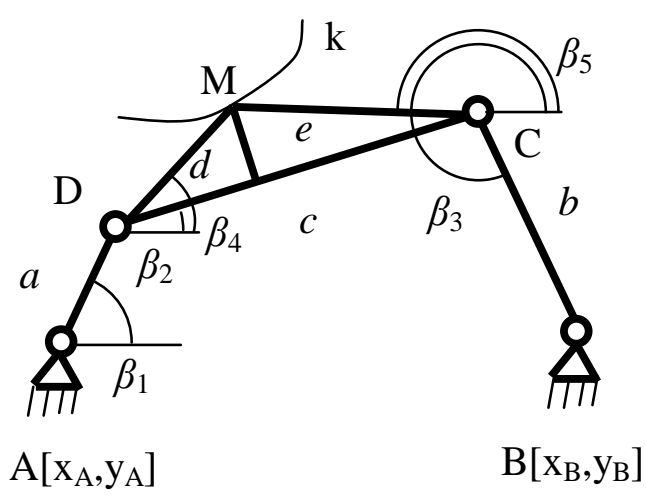

Figure 3: Four-bar mechanism.

where $B_{k}$ are artificially introduced damping coefficients. Dissipation guarantees the removal of the energy from the new dynamic system, and thus brings the system into equilibrium.

The synthesis process is now transformed into the evolution of the accompanying nonlinear dissipative dynamical system. The system has the coordinates $x_{V_{i, r}}, y_{V_{i, r}}$, the kinetic energy (5), the potential energy (6) where the variables are described by formulas (3)-(4) as functions of the coordinates $x_{V_{i, r}}, y_{V_{i, r}}$, the Raleigh function (7) and the initial conditions $x_{V_{i, r}}(0), y_{V_{i, r}}(0)$ as estimations of the positions of the mechanism described by the vector polygon vertices $i=1, \ldots, n$ in particular positions $r=1, \ldots, N$. It is supposed that this new accompanying system reaches its equilibrium given by

$$
E_{\mathrm{k}}=0, \quad E_{\mathrm{p}}=0
$$

and $E_{\mathrm{k}}=0$ results from (5) into

$$
\dot{x}_{k, r}=0, \quad \dot{y}_{k, r}=0
$$

and $E_{\mathrm{p}}=0$ results from (6) into

$$
p_{k, r}=p_{k, i}
$$

These final values $p_{k}=p_{k, r}$ are the synthesized parameters (dimensions) describing the synthesized mechanism [14-17].

\section{Extension of the method}

The method described here deals just with the parametric positional synthesis of a mechanism. The synthesis is usually more complicated, and it can generally be described as the minimization/ maximization of the set of further objective functions

$$
\left\{\begin{array}{l}
\min C F_{\ell}\left(s_{1}, s_{2}, \ldots, s_{n}, p_{1}, p_{2}, \ldots, p_{m}\right), \\
\ell=1,2, \ldots, n_{C F} .
\end{array}\right.
$$

These objective functions are taken into consideration by extending the potential energy (6) by new terms

$$
E_{\mathrm{p}}=\cdots+\frac{1}{2} \sum_{\ell=1}^{n_{C F}} q_{\ell}\left(C F_{\ell}-C F_{\mathrm{D}, \ell}\right)
$$

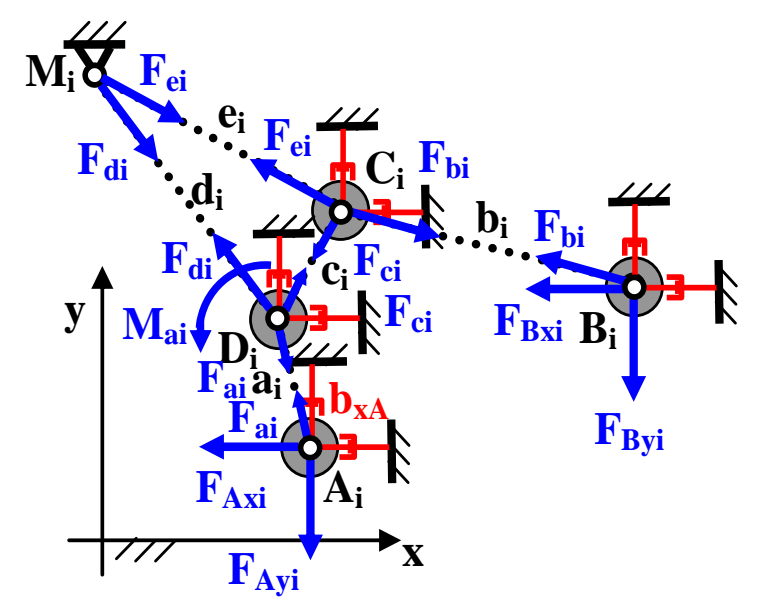

Figure 4: Associated dissipative system of the fourbar mechanism.

where $q_{\ell}$ are chosen positive constants and $C F_{\mathrm{D}, \ell}$ are desired values of the objective function $C F_{\ell}$.

Then the equilibrium (8) of the new accompanying dynamic system also optimizes the objective functions (11) [15-17]. The artificially introduced parameters $m_{k}, k_{k}, B_{k}$ and $q_{\ell}$ can be chosen as arbitrarily positive numbers, but their values influence the dynamics of the synthesis.

\section{Planar example}

The main disadvantages of general optimization methods combined with traditional kinematical synthesis are a high computational cost, extreme growth of the computational complexity with the number of optimized parameters, together with inability to find the solution even though it exists. The following example shows the main advantage of the evolution of the associated dissipative system over general optimization methods, e.g. genetic algorithms.

The comparison of the methods is focused on the synthesis of the kinematical system (a four-bar mechanism) from [12]. This mechanism was chosen because it is relatively simple and it simultaneously consists of 9 dimensional parameters. Moreover, the example can easily be extended with other optimized parameters, e.g. angles of the crank.

The original classical optimization formulation of the example (Fig. 3) is as follows. The kinematical system has $9+n$ dimensions to be synthesized, where $n$ denotes the number of desired positions of the end effector. These dimensions are the length of the crank $a$, the length of the coupler $c$, the length of the follower $b$, and the lengths of the two rods $d$ and $e$ that are connected with the end effector. The other set of optimized dimensions are the $x$ and $y$ positions of the fixed part of the crank $\left(x_{A}, y_{A}\right)$ and the follower $\left(x_{B}, y_{B}\right)$. The last set of parameters to be optimized 

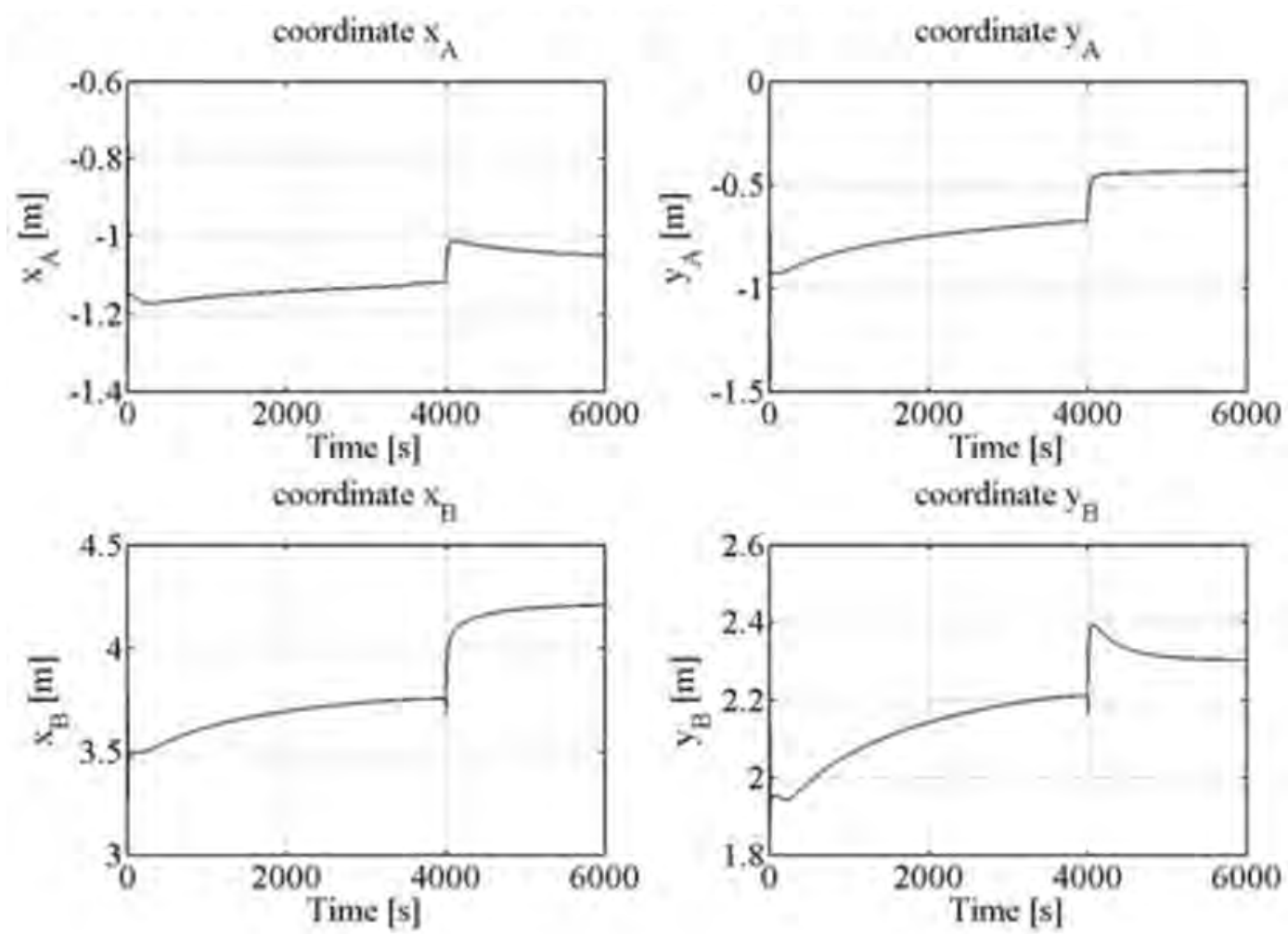

Figure 5: Evolution of the coordinates of a four-bar mechanism.

is the positive increments of the angles $\beta_{1 i}$ of the crank

$$
\beta_{1 i}=\beta_{11}+\sum_{j=2}^{i} \beta_{1 j} .
$$

The point $M$ of the mechanism should pass through the given positions $M_{i}(i=1,2, \ldots, n)$ on the given trajectory.

The coordinates of the mechanism are $\beta_{1}, \beta_{2}$ and $\beta_{3}$. The system constraints are then

$$
\begin{aligned}
a^{2} & =\left(x_{D_{i}}-x_{A_{i}}\right)^{2}+\left(y_{D_{i}}-y_{A_{i}}\right)^{2} \\
b^{2} & =\left(x_{B_{i}}-x_{C_{i}}\right)^{2}+\left(y_{B_{i}}-y_{C_{i}}\right)^{2} \\
c^{2} & =\left(x_{C_{i}}-x_{D_{i}}\right)^{2}+\left(y_{C_{i}}-y_{D_{i}}\right)^{2} \\
d^{2} & =\left(x_{M_{i}}-x_{D_{i}}\right)^{2}+\left(y_{M_{i}}-y_{D_{i}}\right)^{2} \\
e^{2} & =\left(x_{M_{i}}-x_{C_{i}}\right)^{2}+\left(y_{M_{i}}-y_{C_{i}}\right)^{2} .
\end{aligned}
$$

The optimization task is defined as follows

$$
\begin{aligned}
C F & =q_{1} \sum_{i=1}^{n}\left(x_{M i}-x_{M i}^{\prime}\right)^{2} \\
& +q_{2} \sum_{i=1}^{n}\left(y_{M i}-y_{M i}^{\prime}\right)^{2} \rightarrow \min
\end{aligned}
$$

and the set of constraints of the optimization task is

$$
\beta_{1(i+1)}>\beta_{1 i}
$$

where the optimization parameters are: $a, b, c, d, e$, $x_{A}, y_{A}, x_{B}, y_{B}$ and $\beta_{1 i},(i=1,2, \ldots, n)$. The parameters $q_{i}$ again denote the penalization coefficients.

The associated dynamical dissipative system consists of $n$ subsystems for the individual positions of point $M$ (Fig. 4). The masses $m_{A}, m_{B}, m_{D}, m_{E}$ are introduced in the points $A_{i}, B_{i}, D_{i}, E_{i}$. The interactions between the subsystems are ensured by forces of a linear spring nature. A nonzero force acts into the relevant masses whenever the corresponding dimension differs between subsystems $i$ and $j$ $(i, j=1,2, \ldots, n)$. The stabilization of the whole system is ensured by damper elements between the masses and the inertial frame. The constraints of the associated system were changed into the form

$$
\begin{aligned}
a_{i}^{2} & =\left(x_{D_{i}}-x_{A_{i}}\right)^{2}+\left(y_{D_{i}}-y_{A_{i}}\right)^{2}, \\
b_{i}^{2} & =\left(x_{B_{i}}-x_{C_{i}}\right)^{2}+\left(y_{B_{i}}-y_{C_{i}}\right)^{2}, \\
c_{i}^{2} & =\left(x_{C_{i}}-x_{D_{i}}\right)^{2}+\left(y_{C_{i}}-y_{D_{i}}\right)^{2}, \\
d_{i}^{2} & =\left(x_{M_{i}}-x_{D_{i}}\right)^{2}+\left(y_{M_{i}}-y_{D_{i}}\right)^{2}, \\
e_{i}^{2} & =\left(x_{M_{i}}-x_{C_{i}}\right)^{2}+\left(y_{M_{i}}-y_{C_{i}}\right)^{2}, \\
x_{M i} & =\text { const. (prescribed), } \\
y_{M i} & =\text { const. (prescribed). }
\end{aligned}
$$

The forces that act in the dynamical system are as follows

$$
F_{A x i}=\sum_{j=1}^{n} k_{x A}\left(x_{A i}-x_{A j}\right)
$$



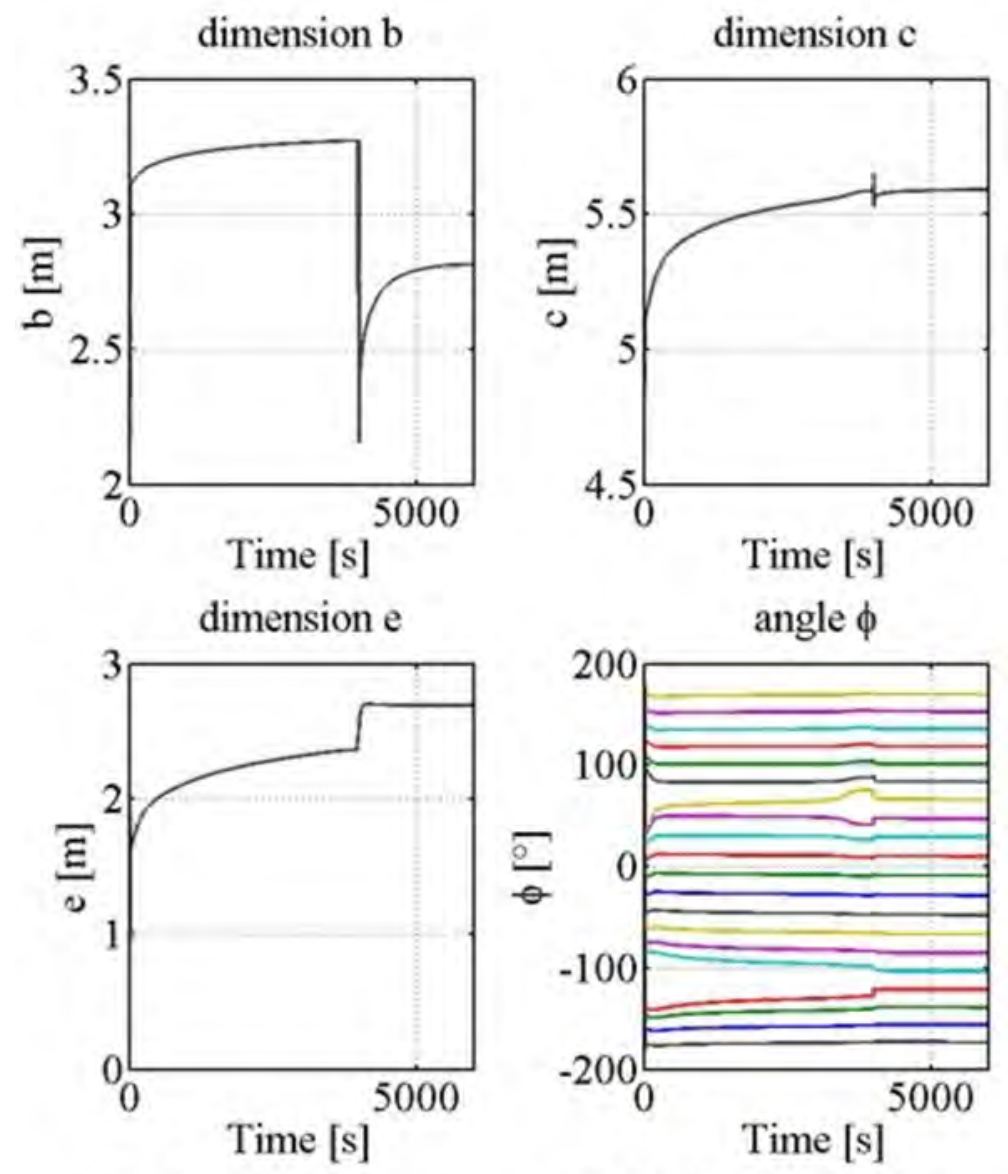

Figure 6: Evolution of the dimensions of a four-bar mechanism.

$$
\begin{aligned}
F_{A y i} & =\sum_{j=1}^{n} k_{y A}\left(y_{A i}-y_{A j}\right), \\
F_{B x i} & =\sum_{j=1}^{n} k_{x B}\left(x_{B i}-x_{B j}\right), \\
F_{B y i} & =\sum_{j=1}^{n} k_{y B}\left(y_{B i}-y_{B j}\right), \\
F_{a i} & =\sum_{j=1}^{n} k_{a}\left(a_{i}-a_{j}\right), \\
F_{b i} & =\sum_{j=1}^{n} k_{b}\left(b_{i}-b_{j}\right), \\
F_{c i} & =\sum_{j=1}^{n} k_{c}\left(c_{i}-c_{j}\right), \\
F_{d i} & =\sum_{j=1}^{n} k_{d}\left(d_{i}-d_{j}\right), \\
F_{e i} & =\sum_{j=1}^{n} k_{e}\left(e_{i}-e_{j}\right), \\
M_{a i} & =k_{M i}\left(\beta_{i-1}-\beta_{i}\right),
\end{aligned}
$$

where for each mechanism position $i$ the equations take into account all the other forces connecting with the other positions $j$ of the mechanism. Therefore the system forms altogether $2 n$ equations. The coefficient $k_{M i}$ takes the nonzero constant value only if $\beta_{1(i+1)}<\beta_{1 i}$. The final dynamical equations for the mass particles in points $\mathrm{B}$ and the algebraic equations that must be fulfilled are as follows

$$
\begin{aligned}
m_{A} \ddot{x}_{A i}= & -\sum_{j=1}^{n} k_{x A}\left(x_{A i}-x_{A j}\right) \\
& +\sum_{j=1}^{n} k_{a}\left(a_{i}-a_{j}\right) \cos \beta_{1 i}-b_{x A} \dot{x}_{A i}, \\
m_{A} \ddot{y}_{A i}= & -\sum_{j=1}^{n} k_{y A}\left(y_{A i}-y_{A j}\right) \\
& +\sum_{j=1}^{n} k_{a}\left(a_{i}-a_{j}\right) \sin \beta_{1 i}-b_{y A} \dot{y}_{A i}, \\
m_{B} \ddot{x}_{B i}= & -\sum_{j=1}^{n} k_{x B}\left(x_{B i}-x_{B j}\right) \\
& -\sum_{j=1}^{n} k_{b}\left(b_{i}-b_{j}\right) \cos \beta_{3 i}-b_{x B} \dot{x}_{B i},
\end{aligned}
$$




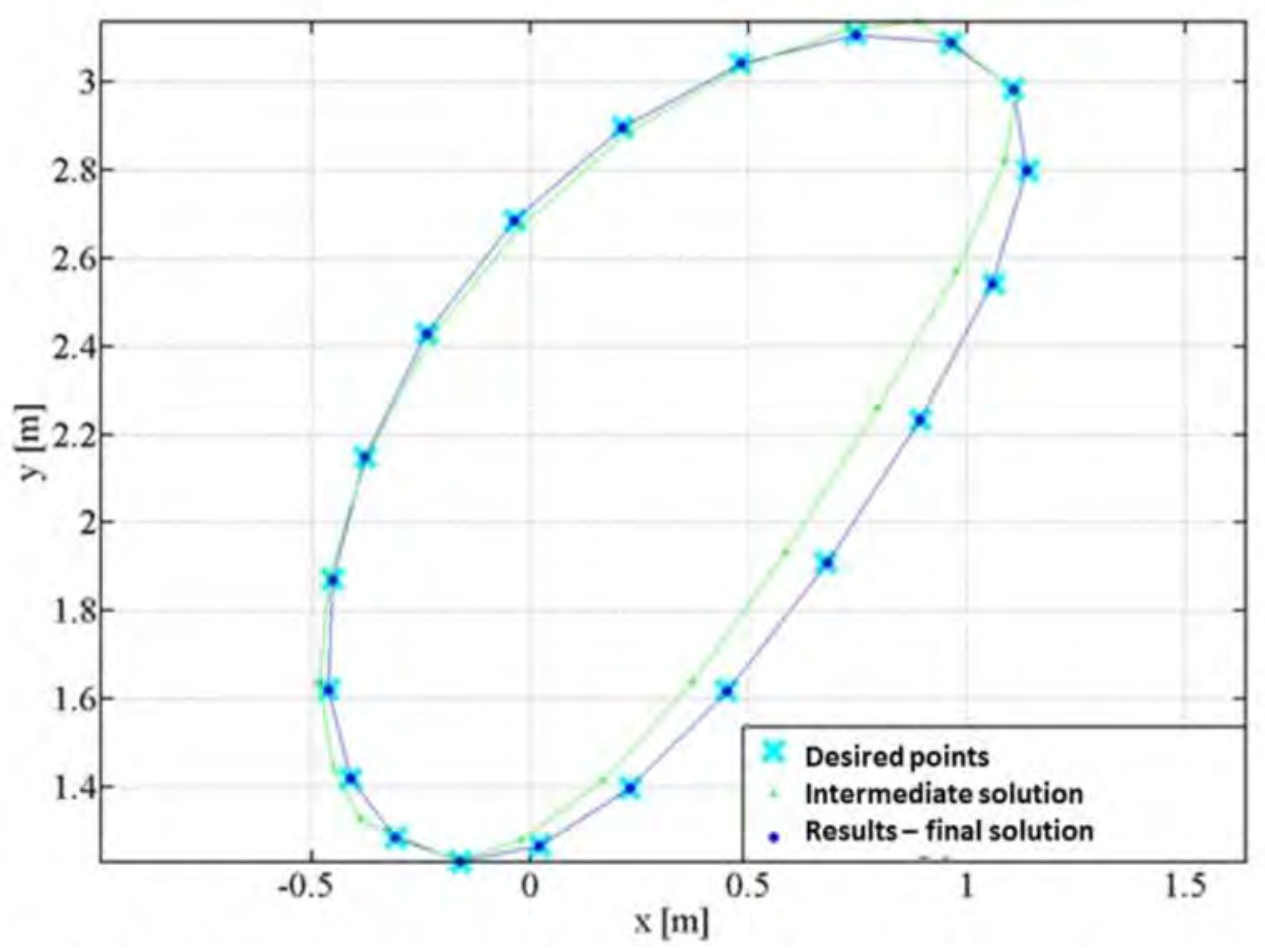

Figure 7: Evolution of the trajectories of a four-bar mechanism by the dissipative system.

$$
\begin{aligned}
m_{B} \ddot{y}_{B i}= & -\sum_{j=1}^{n} k_{y B}\left(y_{B i}-y_{B j}\right) \\
& -\sum_{j=1}^{n} k_{b}\left(b_{i}-b_{j}\right) \sin \beta_{3 i}-b_{y B} \dot{y}_{B i} \\
m_{C} \ddot{x}_{C i}= & \sum_{j=1}^{n} k_{b}\left(b_{i}-b_{j}\right) \cos \beta_{3 i} \\
& -\sum_{j=1}^{n} k_{c}\left(c_{i}-c_{j}\right) \cos \beta_{2 i} \\
& +\sum_{j=1}^{n} k_{e}\left(e_{i}-e_{j}\right) \cos \beta_{5 i}-b_{x C} \dot{x}_{C i} \\
m_{C} \ddot{y}_{C i}= & \sum_{j=1}^{n} k_{b}\left(b_{i}-b_{j}\right) \sin \beta_{3 i} \\
& -\sum_{j=1}^{n} k_{c}\left(c_{i}-c_{j}\right) \sin \beta_{2 i} \\
& +\sum_{j=1}^{n} k_{e}\left(e_{i}-e_{j}\right) \sin \beta_{5 i}-b_{x C} \dot{x}_{C i} \\
m_{D} \ddot{x}_{D i}= & -\sum_{j=1}^{n} k_{a}\left(a_{i}-a_{j}\right) \cos \beta_{1 i} k_{c}\left(c_{i}-c_{j}\right) \cos \beta_{2 i} \\
& k_{d}\left(d_{i}-d_{j}\right) \cos \beta_{4 i}-b_{x D} \dot{x}_{D i}, \\
& +1 \\
& \\
& \\
&
\end{aligned}
$$

$$
\begin{aligned}
m_{D} \ddot{y}_{D i}= & -\sum_{j=1}^{n} k_{a}\left(a_{i}-a_{j}\right) \sin \beta_{1 i} \\
& +\sum_{j=1}^{n} k_{c}\left(c_{i}-c_{j}\right) \sin \beta_{2 i} \\
& +\sum_{j=1}^{n} k_{d}\left(d_{i}-d_{j}\right) \sin \beta_{4 i}-b_{y D} \dot{y}_{D i}
\end{aligned}
$$

where the mechanism dimensions $a_{i}, b_{i}, c_{i}, d_{i}$ and $e_{i}$ are evaluated from the coordinates with the help of the constraint equations formulated in (17).

The simulation started from some selected initial positions $x_{A}, y_{A}, x_{B}, y_{B}, x_{C}, y_{C}, x_{D}$ and $y_{D}$, as was in general described in Section 2, above. The initial positions also determine the initial dimensions $a, b, c$, $d$ and $e$ of the mechanism.

The results of the simulation are presented in the following figures. Fig. 5 and Fig. 6 show the history of the system coordinates. Fig. 7 presents the desired trajectories and the resulting trajectories. The system coordinates $\left(x_{A i}, y_{A i}, x_{B i}, y_{B i}, a_{i}, b_{i}, c_{i}, d_{i}, e_{i}, i=\right.$ $1,2, \ldots, n)$ for all the subsystems (for all the positions) come to rest at the equilibrium values.

The desired trajectory was reached using one reactivation of the dynamic process. All the coordinates for all the subsystems also come to rest at the equilibrium values. These equilibrium values can be interpreted as the searched parameters of the mechanism.

The example was also simulated using genetic algorithms. The boundary conditions for each optimized 


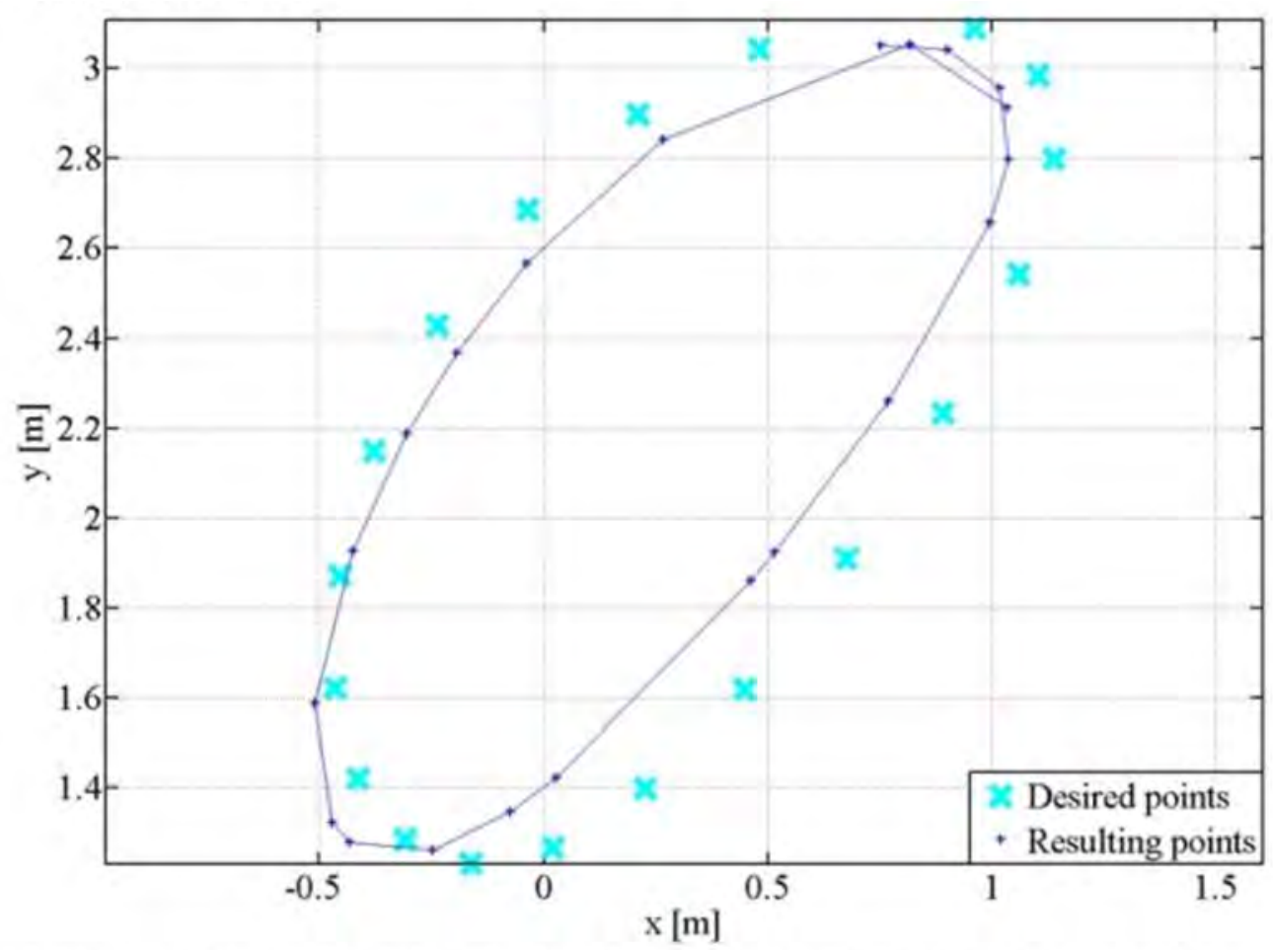

Figure 8: Evolution of the trajectories of a four-bar mechanism using a genetic algorithm.

dimensional parameter were set according to the interval 〈desired, resulting $\rangle$ value of the simulation, which was done by the dissipative system. This interval was extended by $50 \%$ on both sides, and was used as the boundary condition for the particular optimized parameter.

The simulation result is presented in Fig. 8. It is shown that the desired trajectory was not found using this method.

\section{Spatial example}

A further example is the synthesis of a 3D (RSSR) four-bar mechanism [17].

The original classical optimization formulation is presented in Fig. 9. It consists of two skew mechanism axes. The first mechanism axis is identical with the coordinate $y$ axis. The second mechanism is shifted to level $z_{A}$ and rotated by angle $\beta_{A}$ round the $z$ axis. This means that the vector of the axis of the second mechanisms still lies on the plane $x y$. There is a sleeve on the axis of each mechanism. There is a lever on each sleeve. There are spherical linkages on the other side of the levers. At the end, the spherical linkages are connected together by a pitman.

The overall mechanism is naturally described by the height $z_{A}$ of point $A$, the length of the $\ell_{A}$ axis, the angle of rotation $\beta_{A}$, the length of lever $\ell_{A B}$, the length of pitman $\ell_{B D}$, the length of lever $\ell_{C D}$ and the length of the axis $y_{C}=y_{D}$. Let us reformulate the description of the mechanism for conciseness, and in order to avoid the complicated equations in the following formulation.

The mechanism can then be described by the positions $x_{A}, y_{A}, z_{A}$ of the point $A$, by the lengths of the levers $\ell_{A B}$ and $\ell_{C D}$, by the length of the pitman $\ell_{B D}$ and the length of the $y_{D}$ axis. Taking the relevant constraints into account, the representation described here is the minimal representation of the mechanism.

The constraints that describe the mechanism are as follows. The first constraint ensures that points $A$ and $B$ reflect angle $\psi$ for each subsystem. The following constraint ensures that points $C$ and $D$ reflect angle $\varphi$ for each configuration of the mechanism. The third constraint describes the given length of the lever $\ell_{A B}$, and ensures that it is constant across the configurations. The last constraint ensures that vector $\left[x_{A}, y_{A}, 0\right]$ is perpendicular to vector $B A\left(\left[x_{B}, y_{B}, z_{B}\right]-\left[x_{A}, y_{A}, z_{A}\right]\right)$ for each configuration (subsystem). The constraints are

$$
\begin{aligned}
\cos \psi & =\frac{z_{A}-z_{B}}{\sqrt{\left(x_{A}-x_{B}\right)^{2}+\left(y_{A}-y_{B}\right)^{2}+\left(z_{A}-z_{B}\right)^{2}}}, \\
\cos \varphi & =\frac{x_{D}}{x_{D}^{2}+z_{D}^{2}}, \\
\ell_{A B} & =\sqrt{\left(x_{A}-x_{B}\right)^{2}+\left(y_{A}-y_{B}\right)^{2}+\left(z_{A}-z_{B}\right)^{2}} \\
0 & =x_{A}\left(x_{B}-x_{A}\right)+y_{A}\left(y_{B}-y_{A}\right) .
\end{aligned}
$$

Synthesis of the transmission of the RSSR mechanism is a very good example in the sense that in this case the described representation could at the same time mean the set of synthesized parameters. 


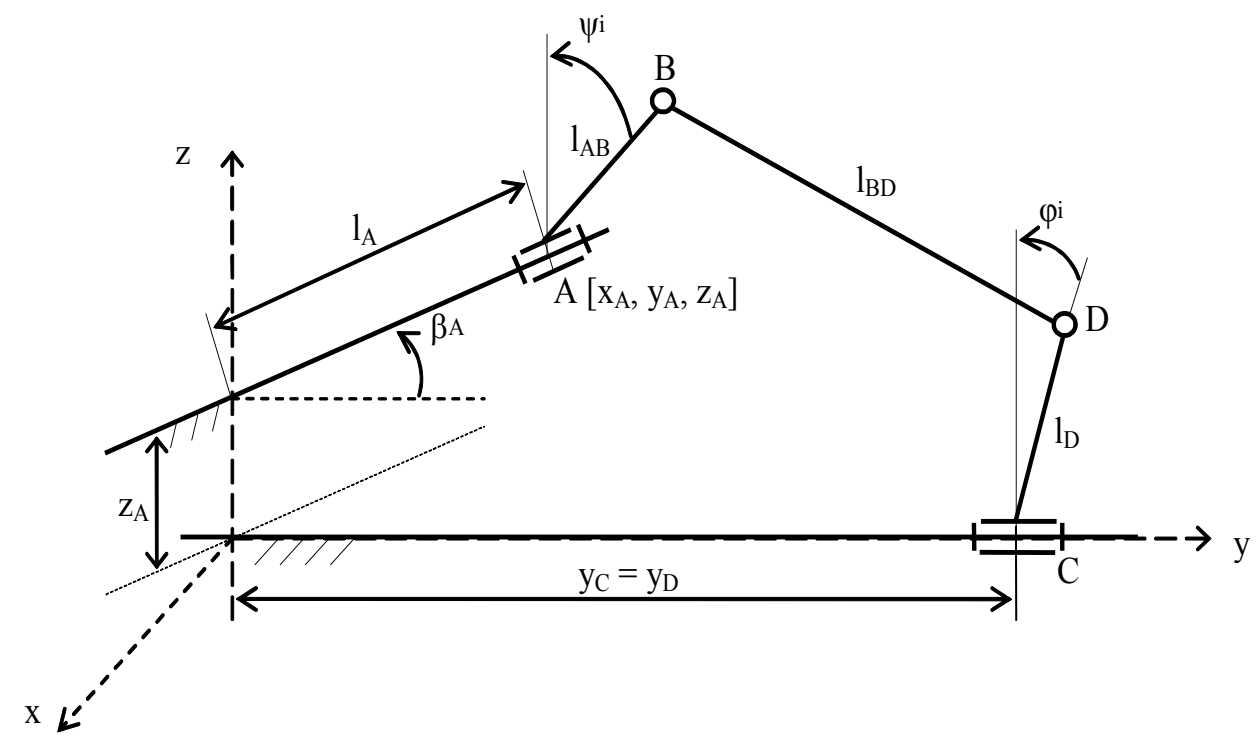

Figure 9: RSSR spatial mechanism.

Because it is a synthesis of transmission with two levers, a global solution of such an example exists and it consists in lengths of the levelers equal to zero. However, a solution of this kind is absolutely unimportant for the practical usability of the synthesized mechanism. Let us therefore choose the length of the lever $\ell_{A B}$ equal to a nonzero constant. The rest of the parameters can then be synthesized. They are once more: $x_{A}, y_{A}, z_{A}, \ell_{B D}, \ell_{C D}, y_{D}$.

The task of transmission synthesis is to find dimensions of the mechanism that fulfill some transmission requirements. The requirement for this example is that the vector of the angles of lever $\varphi$ should correspond with the vector of angles of lever $\psi$. This means that for $\varphi=\varphi_{1}$ is $\psi=\psi_{1}$, for $\varphi=\varphi_{2}$ is $\psi=\psi_{2}$, etc., for the constant dimensions of the mechanism.

To sum up, the optimization task is as follows:

$$
F=\sum_{i=1}^{n}\left(\varphi_{i}-\varphi_{i}^{\prime}\right)^{2} \rightarrow \min
$$

where the optimization parameters are: $x_{A}, y_{A}, z_{A}$, $\ell_{B D}, \ell_{C D}$ and $y_{D}$ and where $\psi_{i}$ is given. Parameters $q_{i}$ again denote the penalization coefficients.

The associated dynamical dissipative system consists of $n$ subsystems for the individual required positions of the transmission mechanism. The masses $m_{A i}, m_{B i}, m_{D i}$ are introduced at points $A_{i}, B_{i}, D_{i}$ and act in the coordinates $x, y, z$. The interactions between the subsystems are ensured by forces of a linear spring nature.

A nonzero force acts into the relevant masses whenever the corresponding dimension differs between subsystems $i$ and $j(i, j=1,2, \ldots, n)$. Stabilization of the whole system is ensured by the damper elements between the masses and the inertial frame, according to the sky-hook idea [20] or [21]. The idea of the transformation is presented in Fig. 10.

In spite of the simple formulation of this example, only using three mass points, the $3 \mathrm{D}$ examples with difficult constraints drive us to formulate the dynamical equations by means of Lagrange equations of mixed type.

The greatest difference that occurs in comparison with simple planar structures is the difficulty of formulating the dynamical system. With simple planar mechanisms, the dynamical equation in direct form can best be formulated using the Newton equations.

The dynamical equations can be defined according to the formulation of the constraints (20). For the sake of conciseness, let us define the lengths of the lever $\ell_{C D}$ and the pitman $\ell_{B D}$, and formulate the $x$, $y, z$ projections of vectors $\left[x_{D}, y_{D}, z_{D}\right]-\left[x_{B}, y_{B}, z_{B}\right]$ and $\left[x_{D}, y_{D}, z_{D}\right]-\left[0, y_{C}, 0\right]$. The lengths are:

$$
\begin{aligned}
& \ell_{D C}=\sqrt{\left(x_{D}-0\right)^{2}+\left(y_{D}-y_{C}\right)^{2}+\left(z_{D}-0\right)^{2}},(22) \\
& \ell_{B D}=\sqrt{\left(x_{D}-x_{B}\right)^{2}+\left(y_{D}-y_{B}\right)^{2}+\left(z_{D}-z_{B}\right)^{2}},
\end{aligned}
$$

The vectors are

$$
\begin{aligned}
& \operatorname{vec}_{x D B}=\frac{x_{B}-x_{B}}{\sqrt{\left(x_{D}-x_{B}\right)^{2}+\left(y_{D}-y_{B}\right)^{2}+\left(z_{D}-z_{B}\right)^{2}}}, \\
& \operatorname{vec}_{y D B}=\frac{y_{B}-y_{B}}{\sqrt{\left(x_{D}-x_{B}\right)^{2}+\left(y_{D}-y_{B}\right)^{2}+\left(z_{D}-z_{B}\right)^{2}}}, \\
& \operatorname{vec}_{z D B}=\frac{z_{B}-z_{B}}{\sqrt{\left(x_{D}-x_{B}\right)^{2}+\left(y_{D}-y_{B}\right)^{2}+\left(z_{D}-z_{B}\right)^{2}}}, \\
& \operatorname{vec}_{x D C}=\frac{x_{D}}{\sqrt{\left(x_{D}-0\right)^{2}+\left(y_{D}-y_{C}\right)^{2}+\left(z_{D}-0\right)^{2}}}, \\
& \operatorname{vec}_{y D C}=0,
\end{aligned}
$$




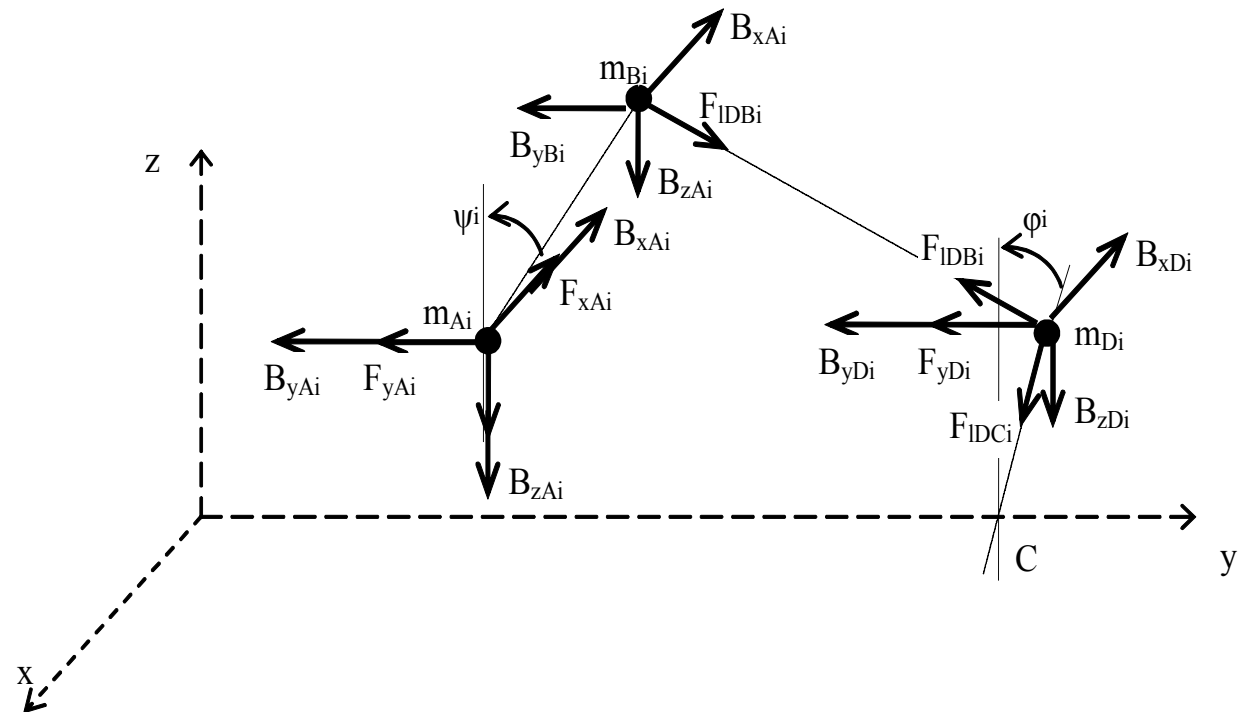

Figure 10: Associated system of the RSSR mechanism.

The lengths (22) and the vectors (23) help in formulating the forces that act in the dynamical system. In addition, the constraints (20) have been changed into the form

$$
\begin{aligned}
\cos \psi & =\frac{z_{B}-z_{A}}{\sqrt{\left(x_{B}-x_{A}\right)^{2}+\left(y_{B}-y_{A}\right)^{2}+\left(z_{B}-z_{A}\right)^{2}}}, \\
\cos \varphi & =\frac{x_{D}}{\sqrt{x_{D}^{2}+z_{D}^{2}}}, \\
\ell_{A B} & =\sqrt{\left(x_{B}-x_{A}\right)^{2}+\left(y_{B}-y_{A}\right)^{2}+\left(z_{B}-z_{A}\right)^{2}}, \\
0 & =x_{A}\left(x_{B}-x_{A}\right)+y_{A}\left(y_{B}-y_{A}\right), \\
\varphi_{i} & =\text { const. } \\
\psi_{i} & =\text { const. }
\end{aligned}
$$

In order to describe the general transmission synthesis of this mechanism, let us take $i=1,2, \ldots, n$ required transmissions and thus $n$ required subsystems of the dynamical system. The dynamical equations and the constraints are identical for each subsystem.

The forces that act in dynamical subsystem $i$ are

$$
\begin{aligned}
F_{x A i} & =\sum_{j=1}^{n} k\left(x_{A i}-x_{A j}\right), \\
F_{y A i} & =\sum_{j=1}^{n} k\left(y_{A i}-y_{A j}\right), \\
F_{z A i} & =\sum_{j=1}^{n} k\left(z_{A i}-z_{A j}\right), \\
F_{\ell D B i} & =\sum_{j=1}^{n} k\left(\ell_{D B i}-\ell_{D B j}\right), \\
F_{\ell D C i} & =\sum_{j=1}^{n} k\left(\ell_{D C i}-\ell_{D C j}\right), \\
F_{y D i} & =\sum_{j=1}^{n} k\left(y_{D i}-y_{D j}\right),
\end{aligned}
$$

$$
\begin{array}{lll}
B_{x A i}=b \dot{x}_{A i}, & B_{y A i}=b \dot{y}_{A i}, & B_{z A i}=b \dot{z}_{A i}, \\
B_{x B i}=b \dot{x}_{B i}, & B_{y B i}=b \dot{y}_{B i}, & B_{z B i}=b \dot{z}_{B i}, \\
B_{x C i}=b \dot{x}_{C i}, & B_{y C i}=b \dot{y}_{C i}, & B_{z C i}=b \dot{z}_{C i} .
\end{array}
$$

This means that for each mechanism position $i$ the equations take into account all the other forces connecting with the other positions $j$ of the mechanism. Therefore the system forms altogether $2 n$ equations.

The mechanism dimensions are evaluated from the coordinates as follows

$$
\begin{aligned}
\ell_{D B i} & =\sqrt{\left(x_{D i}-x_{B i}\right)^{2}+\left(y_{D i}-y_{B i}\right)^{2}+\left(z_{D i}-z_{B i}\right)^{2}}, \\
\ell_{D i} & =\sqrt{x_{D i}^{2}+z_{D i}^{2}} .
\end{aligned}
$$

The final dynamical equations for the mass particles in points $A, B$ and $D$ for dynamical subsystem $i$, together with the algebraic equations, are as follows

$$
\begin{aligned}
m_{A} \ddot{x}_{A i} & =-F_{x A i}-B_{x A i}, \\
m_{A} \ddot{y}_{A i} & =-F_{y A i}-B_{y A i}, \\
m_{A} \ddot{z}_{A i} & =-F_{z A i}-B_{z A i}, \\
m_{B} \ddot{x}_{B i} & =F_{\ell D B i} v e c_{x D B}-B_{x B i}, \\
m_{B} \ddot{y}_{B i} & =F_{\ell D B i} v e c_{y D B}-B_{y B i}, \\
m_{B} \ddot{z}_{B i} & =F_{\ell D B i} \operatorname{vec}_{z D B}-B_{z B i}, \\
m_{D} \ddot{x}_{D i} & =-F_{\ell D B i} v e c_{x D B}-F_{\ell D C i} v e c_{x D C}-B_{x B i}, \\
m_{D} \ddot{y}_{D i} & =-F_{\ell D B i} v e c_{y D B}-F_{y D i}-B_{y B i}, \\
m_{D} \ddot{z}_{D i} & =-F_{\ell D B i} v e c_{z D B}-F_{\ell D C i} v e c_{z D C}-B_{z B i}, \\
\varphi_{i} & =\text { const. } \\
\psi_{i} & =\text { const. }
\end{aligned}
$$

where the integrated coordinates are $\ddot{x}_{A i}, \ddot{y}_{A i}, \ddot{z}_{A i}$, $\ddot{x}_{B i}, \ddot{y}_{B i}, \ddot{z}_{B i}, \ddot{x}_{C i}, \ddot{y}_{C i}, \ddot{z}_{C i}$.

The simulation started from some randomly generated initial positions $x_{A}, y_{A}, z_{A}, x_{B}, y_{B}, z_{B}, x_{D}$, 



Figure 11: Dynamical response of the dimensions of the RSSR mechanism.

$y_{D}, z_{D}$. The initial positions also determine the initial dimensions $x_{A}, y_{A}, z_{A}, \ell_{B D}, \ell_{D C}, y_{D}$, of the mechanism. It is necessary to make a short note on the implementation. All the systems that have been simulated are easy in the context of the formulation.

All the previous dynamical systems were thus formulated by Newton equations. Because this system is already quite complex, Lagrange equations of mixed type were chosen, instead of the Newton equations, as the optimal formulation tool. Thus the formulation was really simplified. However, the great disadvantage of this tool is the instability of the constraints.

The results of the simulation are presented in the following figures. The system coordinates $x_{A i}, y_{A i}, z_{A i}$, $\ell_{B D i}, \ell_{D C i}, y_{D i},(i=1,2, \ldots, 8)$ for all the subsystems come to rest at the equilibrium values (Fig. 11). These equilibrium values can be interpreted as the searched parameters of the mechanism.

The evolution of the constraints is presented in Fig. 12. The red lines show fulfilled constraints. The other lines represent the simulated values of the mechanism. The upper part is dedicated to the given angles $\psi$ and $\varphi$. The simulated values of the angles come to rest at the given equilibrium very soon after the system starts. The lower left picture shows the constraint that ensures the length of the lever $\ell_{A B}$. It can be seen that the given value had been set equal to one. Here, too, the simulated value came to rest at the required value immediately after the system starts. The fourth history presents the perpendicularity of vectors $\left[x_{A}, y_{A}, 0\right]$ and $B A$. This graph, too, shows how soon after the start the constraint condition is fulfilled. It is absolutely clear from all the pictures showing the evolution of the constraint conditions that the required constraints are perfectly fulfilled.

The evolution of the whole structure of the space four-bar mechanism is presented in Fig. 13. The simulation started from the random positions of the structure marked as the initial structure. The simulation finished in the final structure of the mechanism. The final image shows that the corresponding dimensions are equal.

\section{Conclusion}

This paper has described a new method for solving the parametric kinematical synthesis of mechanisms. The robustness and the rapid synthesis procedure of the method have been proven. Especially the robustness is very valuable.

\section{Acknowledgements}

The authors appreciate kind support from the grant MSM6840770003 "Algorithms for computer simulation and application in engineering". 

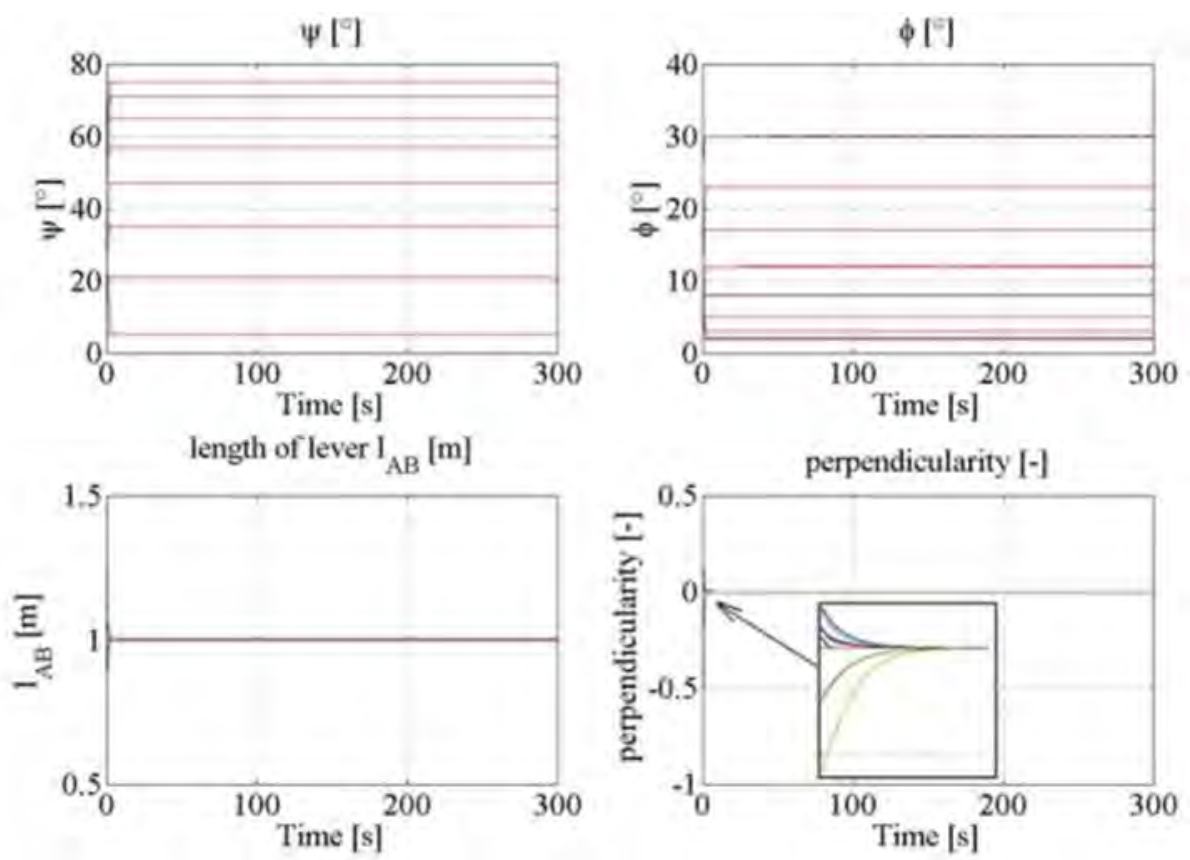

Figure 12: Fulfilling the constraints of the RSSR mechanism.



Structure after $99.76 \mathrm{~s}$

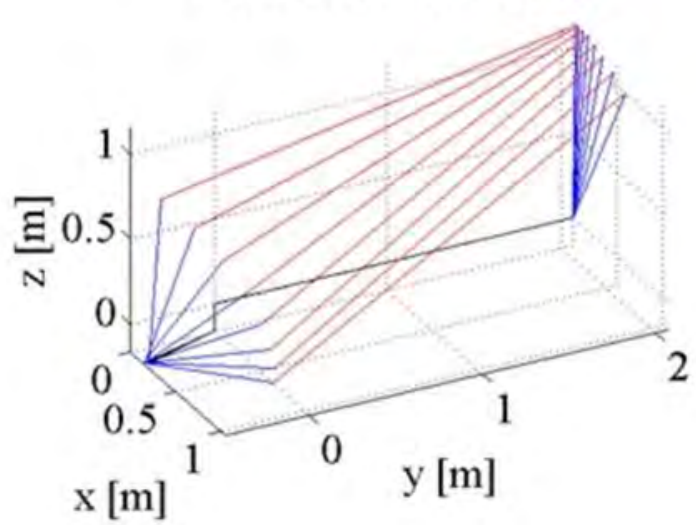

Structure after $199.77 \mathrm{~s}$

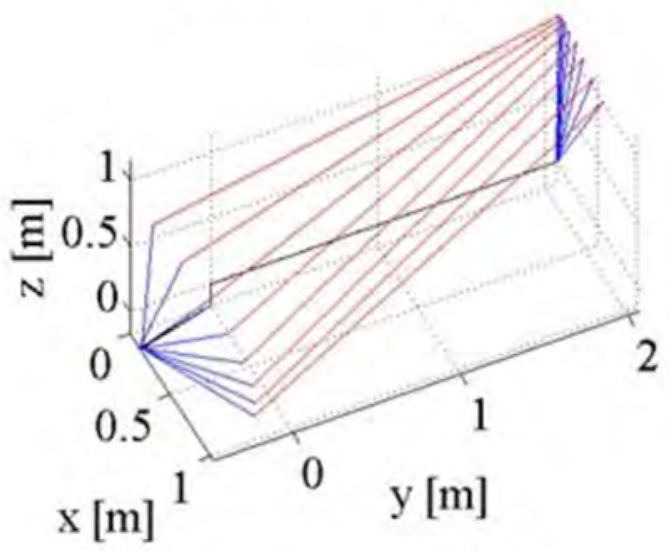

Final Mechanism at $299.77 \mathrm{~s}$

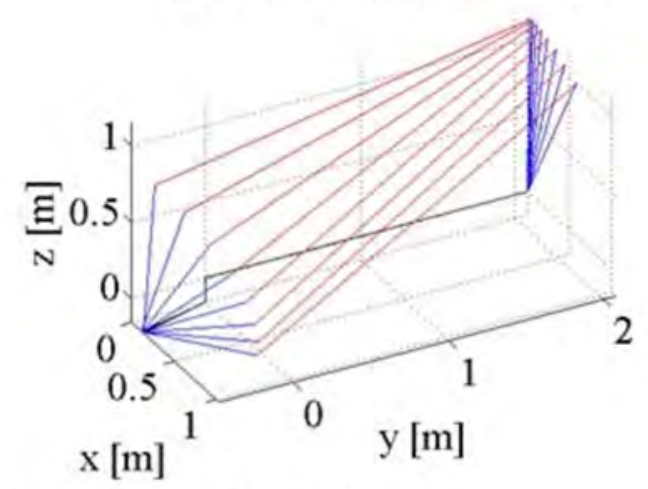

Figure 13: Evolution of the structure of the RSSR mechanism. 


\section{References}

[1] Haug, E.J.: Computer Aided Analysis and Optimization of Mechanical System Dynamics. NATO ASI, Vol. F9, Springer Verlag, Berlin, 1984.

[2] Hartenberg, R.S., Denavit, J.: Kinematic synthesis of linkages, McGraw-Hill, 1964.

[3] Hall, A.S., Jr.: Kinematics and Linkage Design, Balt Publisher, West Lafayette, IN, 1966.

[4] Sandor, G.N. Erdman, A.G.: Advanced Mechanism Design: Analysis and Synthesis, Prentice Hall, 1984.

[5] Erdman, A.G., Sandor, G.N.: Mechanism Design, Analysis and Synthesis, Prentice-Hall, Englewood Cliffs, NJ, 1991.

[6] Hansen, M.R.: A general procedure for dimensional synthesis of mechanisms, Mechanism Design and Synthesis 46, 1992, pp. 67-71.

[7] Bruns, T.: Design of planar, kinematic, rigid body mechanisms, Master's thesis, University of Illinois at Urbana-Champaign, Urbana, IL, USA, 1992.

[8] Hansen, J.M., Tortorelli, D.A.: An efficient method for synthesis of mechanisms, In: D. Beste and W.Schiehlen (eds): Optimization of Mechanical Systems, Kluwer, Dordrecht, 1995, pp. 129-138.

[9] Hansen, M.R., Hansen, J.M.: An efficient method for synthesis of planar multibody systems including shapes of bodies as design variables, Multibody System Dynamics 2, 1998, pp. 115-143.

[10] Paradis, M.J., Willmert, K.D.: Optimal mechanism design using the Gauss constrained method, Journal of Mechanisms, Transmissions, Automation in Design 105, 1983, pp. 187-196.

[11] Minnaar, R.J. et al.: On non-assembly in the optimal dimensional synthesis of planar mechanisms, Structural Optimization 21, 2001, pp. $345-354$.
[12] Hansen, J.M.: Synthesis of Mechanisms Using Time-Varying Dimensions. Multibody System Dynamics, Vol. 7/1, 2002, pp. 127-144.

[13] Jensen, O.F., Hansen, J.M.: Dimensional Synthesis of Spatial Mechanisms and the Problem of Non-Assembly. Multibody System Dynamics, Vol. 15/2, 2006, pp. 107-133.

[14] Valášek, M., Šika, Z.: Mechanism Synthesis as Control Problems. In: Proc. of Workshop on Interaction and Feedbacks '2004, edited by I. Zolotarev, Prague, 2004, pp. 187-192.

[15] Gresl, M. et al.: Synthesis of Dexterity Measure of Mechanisms by Evolution of Dissipative System, Applied and Computational Mechanics, 1 (2007), 2, pp. 461-468.

[16] Sika, Z. et al.: Synthesis of Mechanisms using Evolution of Associated Dissipative Systems, Multibody System Dynamics, 28 (2012), 4, pp. 419-440.

[17] Grešl, M.: Synthesis and Multiobjective Optimization of Mechanisms using Evolution of Associated Dissipative System, Ph.D. Thesis, FME CTU in Prague, Prague, 2010.

[18] Valasek, M.: Multibody Dynamics Without Analytical Mechanics, In: J.A.C. Ambrosio (ed.): Proc. of Int. Conf. on Advances in Computational Multibody Dynamics, IST IDMEC, Lisbon 2003, CD ROM, pp. 1-14.

[19] Stejskal, V., Valasek, M.: Kinematics and Dynamics of Machinery, Marcel Dekker, New York 1996.

[20] Karnopp, D., Crosby, M., Harwood, R.A.: Vibration Control Using Semi-Active Force Generators. Journal of Engineering for Industry, No. 96, 1974, pp. 619-626.

[21] Valášek, M., Kortüm, W., Šika, Z., Magdolen, L., Vaculín, O.: Development of Semi-Active RoadFriendly Truck Suspensions. Control Engineering Practice, Vol. 6, 1998, pp. 735-744. 